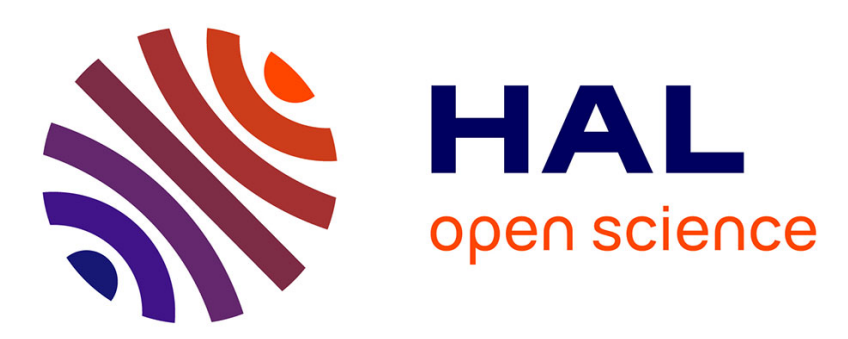

\title{
Homomorphic Characterizations of Indexed Languages
}

Severine Fratani, El Makki Voundy

\section{To cite this version:}

Severine Fratani, El Makki Voundy. Homomorphic Characterizations of Indexed Languages. Language and Automata Theory and Applications (LATA) 2016, Mar 2016, Prague, Czech Republic. 10.1007/978-3-319-30000-9_28. hal-01478780

\section{HAL Id: hal-01478780 \\ https://hal.science/hal-01478780}

Submitted on 6 Mar 2017

HAL is a multi-disciplinary open access archive for the deposit and dissemination of scientific research documents, whether they are published or not. The documents may come from teaching and research institutions in France or abroad, or from public or private research centers.
L'archive ouverte pluridisciplinaire HAL, est destinée au dépôt et à la diffusion de documents scientifiques de niveau recherche, publiés ou non, émanant des établissements d'enseignement et de recherche français ou étrangers, des laboratoires publics ou privés. 


\title{
Homomorphic Characterizations of Indexed Languages
}

\author{
Séverine Fratani and El Makki Voundy \\ Aix-Marseille Université, CNRS, LIF UMR 7279, 13000, Marseille, France
}

\begin{abstract}
We study a family of context-free languages that reduce to $\varepsilon$ in the free group and give several homomorphic characterizations of indexed languages relevant to that family.
\end{abstract}

Keywords: grammars, homomorphic characterizations, transductions, indexed languages

\section{Introduction}

The well known Chomsky-Schützenberger theorem [6] states that every contextfree language $L$ can be represented as $L=h\left(R \cap \mathcal{D}_{k}\right)$, for some integer $k$, regular set $R$ and homomorphism $h$. The set $\mathcal{D}_{k}$ used in this expression, called Dyck language, is the set of well-bracketed words over $k$ pairs of brackets. Combined with Nivat's characterization of rational transductions, this means that any contextfree language can be defined as a set $L=h\left(g^{-1}\left(\mathcal{D}_{2}\right) \cap R\right)$, for some regular set $R$, and homomorphisms $h$ and $g$.

Let us consider wider families of languages, Maslov defines in [13] an infinite hierarchy of languages included in recursively enumerable languages. The level 1 consists of context-free languages, the level 2 of indexed languages (initially defined by Aho [1]). Known as higher order languages since the last decades, the languages of the hierachy and derived objects as higher order trees [12], higher order schemes [9], or higher order graphs [5], are used to model programming languages and are in the core of the recent researches in program verification[18].

It is stated in [14] and proved in [8] that each level $\mathcal{L}_{k}$ of the hierarchy is a principal rational cone generated by a language $M_{k} \in \mathcal{L}_{k}$. This means that each language in $\mathcal{L}_{k}$ is the image of $M_{k}$ by a rational transduction. Roughly speaking, the language $M_{k}$ consists of words composed by $k$ embedded Dyck words and can be viewed as a generalization of the Dyck language. Indeed it gives a description of derivations of an indexed grammar of level $k$, in the same way that the Dyck language encodes derivations of a context-free grammar.

This latter characterization describes $\mathcal{L}_{k}$ from a single language $M_{k}$, but this one is very complicated as soon as $k \geq 2$, as the majority of higher order languages. To better understand higher order languages, we think that it is necessary to characterize them using more simple objects. So, we may wonder whether it is possible to give versions of the Chomsky-Schützenberger theorem and a characterization by transduction of the level $k+1$ of the hierarchy, using 
only the level $k$ of the hierarchy. The fundamental point is then to identify mechanisms that bridge the level $k$ to the level $k+1$.

In this paper, we solve the problem for the class IL of Indexed Languages (the level 2 of the hierarchy). In order to localize the problem, let us remark that from [10], recursively enumerable languages are sets that can be written as $L=h\left(K \cap \mathcal{D}_{k}\right)$ where $K$ is a context-free language, and $h$ a homomorphism. So if we want a homomorphic characterization of IL using only context-free or regular languages, we would have to consider a restricted class of context-free languages.

For this purpose, we introduce the class of $\varepsilon$-Reducible Context-Free Languages $(\varepsilon$-CFLs), which is a strict subclass of context-free languages that reduce to $\varepsilon$ by the bilateral reduction $S=\{a \bar{a} \rightarrow \varepsilon, \bar{a} a \rightarrow \varepsilon\}_{a \in \Gamma}$ (these languages are thus defined over an alphabet $\Gamma$ and its copy $\bar{\Gamma}$ ). We extend this definition to transductions: an $\varepsilon$-Reducible Context-Free Transduction $(\varepsilon$-CFT) is a contextfree transduction whose domain is an $\varepsilon$-CFL. Using these objects, we obtain simple and elegant generalizations of the Chomsky-Schützenberger theorem. Indexed languages are:

- the images of $\mathcal{D}_{2}$ by $\varepsilon$-reducible context-free transductions. (Theorem 15);

- sets $h\left(Z \cap \mathcal{D}_{k}\right)$; where $k$ is an integer, $Z$ an $\varepsilon$-CFL, and $h$ a homomorphism

(Theorem 18).

Beyond these two results, we study the classes of $\varepsilon$-CFLs and $\varepsilon$-CFTs defined by means of context-free grammars and context-free transduction grammars. First we express them using symmetric homomorphisms which are homomorphisms under which there are closed. We establish a Chomsky-Schützenbergerlike Theorem for $\varepsilon$-CFLs, and a Nivat-like characterization for $\varepsilon$-CFTs: every $\varepsilon$-CFL $L$ can be represented as $L=g\left(R \cap \mathcal{D}_{k}\right)$ for some integer $k$, regular language $R$, and symmetric homomorphism $g$; and $\varepsilon$-CFTs are relations that can be represented as $\left\{(g(x), h(x)) \mid x \in R \cap \mathcal{D}_{k}\right\}$ for some integer $k$, regular language $R$, homomorphism $h$ and symmetric homomorphism $g$. This leads to a third characterization: indexed languages are languages that can be described as $L=h\left(g^{-1}\left(\mathcal{D}_{2}\right) \cap R \cap \mathcal{D}_{k}\right)$, for some integer $k$, regular language $R$, homomorphism $h$ and symmetric homomorphism $g$ (Corollary 17 ).

Similar characterizations have been given for subclasses of indexed languages, by Weir [20] for linear indexed languages, by Kanazawa [11] and Sorokin [19] for yields of tree languages generated by simple context-free grammars. The main difference is that in their cases, the homomorphism $g$ is not symmetric, but is fixed in function of $k$.

Overview. Section 1 is devoted to the study of $\varepsilon$-CFLs. After introducing necessary notions as free groups and Dyck languages, we define the class of grammars generating $\varepsilon$-CFLs. We then study their closure properties, and conclude the section by giving a Chomsky-Schützenberger-like characterization of the class of $\varepsilon$-CFLs. In Section 2, we extend our definition to transductions and define the class of $\varepsilon$-CFTs. After a subsection giving background on transductions we 
give a Nivat-like characterization of $\varepsilon$-CFTs. The last section is devoted to indexed languages. After introducing indexed grammars, we prove that indexed languages are images of the Dyck language by $\varepsilon$-CFTs and deduce from this result several homomorphic characterizations.

\section{Epsilon-Reducible Context-Free Languages}

In this section, we study a family of context-free languages defined over a union of an alphabet and its opposite-disjoint copy and that reduce to the neutral element $\varepsilon$ when projected into the free group. The main result here is a ChomskySchützenberger-like homomorphic characterization of these languages. We assume the reader to be familiar with context-free grammars and languages (see [3] for example), and present below a few necessary notions on free groups.

\subsection{Free groups and Dyck languages}

Given an alphabet $\Gamma$, we denote by $\bar{\Gamma}$ a disjoint copy $\bar{\Gamma}=\{\bar{a} \mid a \in \Gamma\}$ of it, and by $\widehat{\Gamma}$ the set $\Gamma \cup \bar{\Gamma}$. We adopt the following conventions: $\overline{\bar{a}}=a$ for all $a \in \Gamma$, $\bar{\varepsilon}=\varepsilon$ and for any word $u=\alpha_{1} \cdots \alpha_{n} \in \widehat{\Gamma}^{*}, \bar{u}=\overline{\alpha_{n}} \cdots \overline{\alpha_{1}}$.

Let us consider the reduction system $S=\{(a \bar{a}, \varepsilon),(\bar{a} a, \varepsilon)\}_{a \in \widehat{\Gamma}}$. A word in $\widehat{\Gamma}^{*}$ is said to be reduced if it is $S$-reduced, i.e. it does not contain occurrences of $a \bar{a}, \bar{a} a$, for $a \in \Gamma$. As $S$ is confluent, each word $w$ is equivalent $\left(\bmod \leftrightarrow \leftrightarrow_{S}^{*}\right)$ to a unique reduced word denoted $\rho(w)$. Note that for all $u \in \widehat{\Gamma}^{*}, \rho(u \bar{u})=\rho(\bar{u} u)=\varepsilon$. Given a set $X$, we denote by $\rho(X)$ the set $\{\rho(x) \mid x \in X\}$.

The free group $\mathrm{F}(\Gamma)$ consists of reduced words over $\widehat{\Gamma}$. Its neutral element is the empty word and its product $\bullet$ is defined as $u \bullet v=\rho(u v)$.

The set of all words $u \in \widehat{\Gamma}^{*}$ such that $\rho(u)=\varepsilon$ is denoted $\mathcal{T}_{\Gamma}$. The Dyck language over $\Gamma$, denoted $\mathcal{D}_{\Gamma}$, is the set of all $u \in \mathcal{T}_{\Gamma}$, such that for every prefix $v \preccurlyeq u$ : $\rho(v) \in \Gamma^{*}$. We will also write $\mathcal{D}_{k}, k \geq 1$, to refer to the set of Dyck words over any alphabet of size $k$.

\section{$2.2 \varepsilon$-Reducible Context-Free Languages and Grammars}

Definition 1. An $\varepsilon$-Reducible Context-Free Grammar $(\varepsilon-C F G)$ is a context free grammar $G=(N, T, S, P)$ ( $N$ is the set of nonterminal symbols, $\widehat{\Gamma}$ the terminal alphabet, $S \in N$ is the start symbol, and $P$ is the set of productions) such that $T=\widehat{\Gamma}$ for some alphabet $\Gamma$ and every production is in the form:

$$
X \longrightarrow \omega \Omega \bar{\omega}, \quad \text { for } \omega \in \widehat{\Gamma}^{*}, \text { and } \Omega \in N^{*}
$$

For all $X \in N$, we define $\mathcal{L}_{G}(X)=\left\{u \in \widehat{\Gamma}^{*} \mid X \stackrel{*}{\longrightarrow}_{G} u\right\}$; the language generated by $G$ is $\mathcal{L}_{G}=\mathcal{L}_{G}(S)$.

An $\varepsilon$-Reducible Context-Free Language $(\varepsilon-C F L)$ is a context-free language $L$ that can be generated by an $\varepsilon-C F G$. 
Example 2. Let $G=(N,\{\alpha, \beta, \bar{\alpha}, \bar{\beta}\}, S, P)$ be the $\varepsilon$-CFG whose productions are: $S \longrightarrow \beta X \bar{\beta}, X \longrightarrow \alpha X \bar{\alpha}+Y, Y \longrightarrow \bar{\alpha} Y Z \alpha+\bar{\beta} \beta, Z \longrightarrow \bar{\alpha} Z \alpha+\bar{\beta} \beta$.

One can easily check that:

$$
\begin{array}{ll}
\mathcal{L}_{G}(Z)=\bigcup_{n \geq 0} \bar{\alpha}^{n} \bar{\beta} \beta \alpha^{n}, & \mathcal{L}_{G}(Y)=\bigcup_{n \geq 0} \bar{\alpha}^{n} \beta \bar{\beta}\left(\Pi_{i=1}^{n} \mathcal{L}_{G}(Z) \alpha\right), \\
\mathcal{L}_{G}(S)=\beta \mathcal{L}_{G}(X) \bar{\beta}, & \mathcal{L}_{G}(X)=\bigcup_{n \geq 0} \alpha^{n} \mathcal{L}_{G}(Y) \bar{\alpha}^{n} .
\end{array}
$$

It follows that: $\mathcal{L}_{G}=\bigcup_{n, m, r_{1}, \ldots . r_{m} \geq 0} \beta \alpha^{n} \bar{\alpha}^{m} \bar{\beta} \beta\left(\Pi_{i=1}^{m} \bar{\alpha}^{r_{i}} \bar{\beta} \beta \alpha^{r_{i}+1}\right) \bar{\alpha}^{n} \bar{\beta}$.

It seems clear that every $\varepsilon$-CFL $L$ satisfies $\rho(L)=\{\varepsilon\}$. One can indeed observe that every terminal word generated from a nonterminal symbol $X \in N$ reduce to $\varepsilon$. However, there are context-free languages that reduce to $\varepsilon$ and which cannot be generated by an $\varepsilon$-CFG. We prove this by using a "pumping lemma" for $\varepsilon$-CFLs.

Lemma 3. If $L \subseteq \widehat{\Gamma}^{*}$ is an $\varepsilon$-CFL, then there exists some integer $p \geq 1$ such that every word $s \in L$ with $|s| \geq p$ can be written as $s=u v w x y$ with

1. $\rho(u y)=\varepsilon, \rho(v x)=\varepsilon$ and $\rho(w)=\varepsilon$

2. $|v w x| \leq p$,

3. $|v x| \geq 1$, and

4. $u v^{n} w x^{n} y$ is in $L$ for all $n \geq 0$.

Proof (Sketch). Let $G$ be an $\varepsilon$-CFG generated $L$. The proof of the pumping lemma for context-free languages is based on the fact that if a word $s \in L$ is long enough, there are a non terminal $A$ and terminal words $u, v, w, x, y$ such that $S \stackrel{*}{\longrightarrow}_{G} u A y \stackrel{*}{\longrightarrow}_{G} u v A x y \stackrel{*}{\longrightarrow}_{G} u v w x y$ and $s=u v w x y$. Since $G$ is an $\varepsilon$-CFG, this implies that $\rho(u y)=\varepsilon, \rho(v x)=\varepsilon$ and $\rho(w)=\varepsilon$

Proposition 4. There is a context-free language $L$ satisfying $\rho(L)=\varepsilon$ which is not an $\varepsilon-C F L$.

Proof (Sketch). By applying Lemma 3 to the set $L$ of words $(\alpha \bar{\alpha})^{n} \beta(\alpha \bar{\alpha})^{n} \bar{\beta}$ for $n \geq 0$, we can show that $L$ is not an $\varepsilon$-CFL.

Proposition 5. The class of $\varepsilon$-CFLs is closed under union, intersection with regular sets, concatenation and Kleene star.

Proof. Obviously, the class of $\varepsilon$-CFLs is closed under union, concatenation and Kleene star. Let us prove the closure under intersection with regular sets. Let $L$ be generated by an $\varepsilon$-CFG $G=(N, \widehat{\Gamma}, P, S)$ and $R$ be a regular language. There is a monoid morphism $\mu: \widehat{\Gamma}^{*} \rightarrow M$, where $M$ is a finite monoid and $H \subseteq M$ such that $R=\mu^{-1}(H)$. We construct the $\varepsilon$-CFG $G^{\prime}=\left(N^{\prime}, \widehat{\Gamma}, P^{\prime}, S^{\prime}\right)$ where $N^{\prime}=\left\{X_{m} \mid X \in N, m \in M\right\} \cup\left\{S^{\prime}\right\}$ and $P^{\prime}$ is the set of all productions:

$-X_{m} \longrightarrow \alpha X_{1, m_{1}} \cdots X_{n, m_{n}} \bar{\alpha}$ such that $X \longrightarrow \alpha X_{1} \cdots X_{n} \bar{\alpha} \in P$ and $m=$ $\mu(\alpha) m_{1} \cdots m_{n} \mu(\bar{\alpha})$ 
$-S^{\prime} \longrightarrow S_{m}$ for $m \in H$

Then for every $u \in \widehat{\Gamma}^{*}$, for every $X \in N$ and $m \in M$ :

$$
X_{m} \stackrel{*}{\longrightarrow}_{G^{\prime}} u \text { iff } X \stackrel{*}{\longrightarrow} G u \text { and } u \in \mu^{-1}(m) .
$$

It follows that $\mathcal{L}_{G^{\prime}}=L \cap \mu^{-1}(H)$.

\subsection{A Chomsky-Schützenberger-like theorem for $\varepsilon$-CFLs}

The Chomsky-Schützenberger theorem states that a language $L \subseteq \Sigma^{*}$ is contextfree iff there is an alphabet $\Gamma$, a regular set $R \subseteq \widehat{\Gamma}^{*}$, and a homomorphism $h: \widehat{\Gamma}^{*} \rightarrow \Sigma^{*}$ such that

$$
L=h\left(R \cap \mathcal{D}_{\Gamma}\right) .
$$

This implies that the whole class of context-free languages is generated by homomorphic images of $\varepsilon$-CFLs, since $R \cap \mathcal{D}_{B}$ is an $\varepsilon$-CFL. To get an homomorphic characterization for $\varepsilon$-CFLs, we introduce a class of homomorphisms under which the family of $\varepsilon$-CFLs is closed.

Definition 6. A homomorphism $g: \widehat{\Sigma}^{*} \rightarrow \widehat{\Gamma}^{*}$ is said to be symmetric if for all $\alpha \in \widehat{\Sigma}, g(\bar{\alpha})=\overline{g(\alpha)}$.

Proposition 7. The class of $\varepsilon-C F L$ s is closed under symmetric homomorphism.

Proof. Consider a language $L$ generated by an $\varepsilon$-CFGG $=(N, \widehat{\Gamma}, P, S)$ and $g: \widehat{\Gamma}^{*} \rightarrow \widehat{\Sigma}^{*}$ be a symmetric homomorphism. We construct an $\varepsilon$-CFGG $G^{\prime}=$ $\left(N, \widehat{\Sigma}, P^{\prime}, S\right)$ generating $g(L)$ as follows:

$$
P^{\prime}=\left\{X \longrightarrow g(u) \Omega g(\bar{u}) \mid X \longrightarrow u \Omega \bar{u} \in P, \Omega \in N^{*}, u \in \widehat{\Gamma}^{*}\right\} .
$$

More generally, $\varepsilon$-CFLs are closed under every homomorphism $g$ satisfying " $\rho(u)=$ $\varepsilon \Longrightarrow \rho(g(u))=\varepsilon "$.

We can now state the main result of this section.

Theorem 8. A set $L \subseteq \widehat{\Gamma}^{*}$ is an $\varepsilon$-CFL iff there is an alphabet $\Sigma$, a symmetric homomorphism $g: \widehat{\Sigma}^{*} \rightarrow \widehat{\Gamma}^{*}$, and a regular set $R \subseteq \widehat{\Sigma}^{*}$ such that

$$
L=g\left(R \cap \mathcal{D}_{\Sigma}\right) .
$$

The "if" part of Theorem 8 is direct using Propositions 5 and 7. The "only if" part is obtained using a slight adaptation of the proof of the non-erasing variant of the Chomsky-Schützenberger theorem given in [17].

We conclude this section by emphasizing that Theorem 8 and Propositions 5 and 7 provide another characterization of the class of $\varepsilon$-CFLs:

Corollary 9. The family of $\varepsilon$-CFLs is the least family of languages that contains the Dyck language and is closed under union, intersection with regular sets, symmetric homomorphisms, concatenation and Kleene star. 


\subsection{Related works}

In [4], the authors define (pure) balanced grammars that are context-free grammars whose set of productions is a (possibly infinite) regular set of rules of the form $X \longrightarrow \alpha m \bar{\alpha}$, where $\alpha \in \Gamma$ and $m \in N^{*}$. Balanced grammars do not generate all $\varepsilon$-CFLs included in $\mathcal{D}_{\Gamma}$, for example they cannot generate the set $\left\{\beta(\alpha \bar{\alpha})^{n}(\gamma \bar{\gamma})^{n} \bar{\beta} \mid n \geq 0\right\}$.

Introduced in [15], input-driven languages, more recently known as Visibly Pushdown Languages (VPLs), are extensions of balanced languages defined over a structured alphabet: $\Sigma_{c}$ is the set of call symbols, $\Sigma_{r}$ the set of returns and $\Sigma_{\ell}$ the set of local symbols. They are recognized by pushdown automata that push onto the stack only when reading a call, pop the stack only on returns, and do not use the stack when reading local actions. The input word hence controls the permissible operations on the stack-however, there is no restriction on the symbols that can be pushed or popped. This implies that there are visibly pushdown languages which are not $\varepsilon$-CFLs. However the $\varepsilon$-CFL $\left\{(\alpha \bar{\alpha})^{n}(\beta \bar{\beta})^{n} \mid\right.$ $n \geq 0\}$ is not a VPL when $\Sigma_{c}=\Gamma$ and $\Sigma_{r}=\bar{\Gamma}$.

Also note that unlike $\varepsilon$-CFLs, VPLs are closed under intersection. We will see (Theorem 18) that the intersection of an $\varepsilon$-CFL with the Dyck language is an indexed language.

\section{Epsilon-Reducible Context-Free Transductions}

In this section, we extend the notion of $\varepsilon$-reducibility to transductions. We consider a subclass of context-free transductions such that their domains are $\varepsilon$-CFLs. We give a Nivat-like presentation of those transductions.

\subsection{Transductions}

We briefly introduce rational and context-free transductions. The reader can refer to [2] for a more detailed presentation.

Let $\Gamma$ and $\Sigma$ be two finite alphabets, we consider the monoid $\Gamma^{*} \times \Sigma^{*}$ whose product is the product on words, extended to pairs of words : $\left(u_{1}, v_{1}\right)\left(u_{2}, v_{2}\right)=$ $\left(u_{1} u_{2}, v_{1} v_{2}\right)$. A subset $\tau$ of $\Gamma^{*} \times \Sigma^{*}$ is called a $(\Gamma, \Sigma)$-transduction.

Transductions are viewed as (partial) functions from $\Gamma^{*}$ toward subsets of $\Sigma^{*}$ : for any $u \in \Gamma^{*}, \tau(u)=\left\{v \in \Sigma^{*} \mid(u, v) \in \tau\right\}$. For every $L \subseteq \Gamma^{*}$, the image (or transduction) of $L$ by $\tau$ is $\tau(L)=\bigcup_{u \in L} \tau(u)$. The domain of $\tau$ is $\operatorname{Dom}(\tau)=\{u \mid \exists v,(u, v) \in \tau\}$.

Rational transductions: A rational $(\Gamma, \Sigma)$-transduction is a rational subset of the monoid $\Gamma^{*} \times \Sigma^{*}$. Among the different characterizations of rational transductions, let us cite the Nivat theorem [16] stating that rational transductions are relations $\tau=\{(g(u), f(u)) \mid u \in R\}$, for some regular set $R$ and homomorphisms $f$ and $g$.

Rational transductions are closed by composition and many classes of languages are closed under rational transductions. In particular, $\tau(L)$ is rational if $L$ is rational, and $\tau(L)$ is context-free if $L$ is context-free. 
Associated with the Nivat theorem, the Chomsky-Schützenberger theorem establish in a stronger version that a language $L$ is context-free iff there is a rational transduction $\tau$ such that $L=\tau\left(\mathcal{D}_{2}\right)$.

Context-free transductions: Following [2, page 62], a transduction $\tau \subseteq \Gamma^{*} \times \Sigma^{*}$ is context-free if there is an alphabet $A$, a context-free language $K \subseteq A^{*}$ and two homomorphisms $f: A^{*} \rightarrow \Sigma^{*}$ and $g: A^{*} \rightarrow \Gamma^{*}$ such that $\tau=\{(g(u), f(u)) \mid$ $u \in K\}$.

Equivalently, $\tau$ is context-free if it is generated by a context-free transduction grammar. This is a context-free grammar whose terminals are pairs of words. Derivations are done as usually but the product used on terminal pairs is the product of the monoid $\Gamma^{*} \times \Sigma^{*}$.

Context-free transductions enjoy however fewer good properties, in particular, [2, page 62] they are not closed under composition and classes of languages are usually not closed under them. For example, images of regulars languages are context-free languages and images of context-free languages are recursively enumerable languages.

\section{$3.2 \varepsilon$-Reducible Context-Free Transductions and Transducers}

Definition 10. An $\varepsilon$-Reducible Context-Free Transduction Grammar $(\varepsilon-C F T G)$ is a context-free transducer $G=(N, \widehat{\Gamma}, \Sigma, S, P)$ in which every production is in the form

$$
X \longrightarrow(\omega, u) \Omega(\bar{\omega}, v), \quad \text { with } \omega \in \widehat{\Gamma}^{*}, u, v \in \Sigma^{*}, \Omega \in N^{*} .
$$

The transduction generated by $G$ is $\mathcal{T}_{G}=\left\{(u, v) \in \widehat{\Gamma}^{*} \times \Sigma^{*} \mid S \stackrel{*}{\longrightarrow}{ }_{G}(u, v)\right\}$. An $\varepsilon$-reducible context-free transduction $(\varepsilon-C F T)$ is a context-free transduction generated by an $\varepsilon-C F T G$.

Example 11. Let $G=(N,\{\alpha, \beta, \bar{\alpha}, \bar{\beta}\},\{a\}, S, P)$ be the $\varepsilon$-CFTG whose productions are:

$$
\begin{aligned}
& S \longrightarrow(\beta, \varepsilon) X(\bar{\beta}, \varepsilon) \quad X \longrightarrow(\alpha, \varepsilon) X(\bar{\alpha}, \varepsilon) X \longrightarrow(\varepsilon, \varepsilon) Y(\varepsilon, \varepsilon) \\
& Y \longrightarrow(\bar{\alpha}, a) Y Z(\alpha, \varepsilon) Z \longrightarrow(\bar{\alpha}, a) Z(\alpha, a) Y \longrightarrow(\bar{\beta}, \varepsilon)(\beta, \varepsilon) Z \longrightarrow(\bar{\beta}, \varepsilon)(\bar{\beta}, \varepsilon) .
\end{aligned}
$$

Let $\tau$ be the transduction generated by $G$. The domain of $\tau$ is the $\varepsilon$-CFL given in Example 2 and one can easily check that

$$
\tau=\bigcup_{n, m, r_{1}, \ldots r_{m} \geq 0}\left(\beta \alpha^{n} \bar{\alpha}^{m} \bar{\beta} \beta\left(\Pi_{i=1}^{m} \bar{\alpha}^{r_{i}} \bar{\beta} \beta \alpha^{r_{i}+1}\right) \bar{\alpha}^{n} \bar{\beta}, a^{m+2 r_{1}+\cdots+2 r_{m}}\right) .
$$

Theorem 12. Given a transduction $\tau \subseteq \widehat{\Gamma}^{*} \times A^{*}$, the following properties are equivalent:

1. $\tau$ is an $\varepsilon$-reducible context-free transduction; 
2. there is an alphabet $\Delta$, an $\varepsilon-C F L X \subseteq \widehat{\Delta}^{*}$, a symmetric homomorphism $g: \widehat{\Delta}^{*} \rightarrow \widehat{\Gamma}^{*}$ and a homomorphism $h: \widehat{\Delta}^{*} \rightarrow A^{*}$ such that

$$
\tau=\{(g(u), h(u)) \mid u \in X\}
$$

3. there is an alphabet $\Delta$, a symmetric homomorphism $g: \widehat{\Delta}^{*} \rightarrow \widehat{\Gamma}^{*}$, a homomorphism $h: \widehat{\Delta}^{*} \rightarrow A^{*}$ and a regular set $R \subseteq \widehat{\Delta}^{*}$ such that

$$
\tau=\left\{(g(u), h(u)) \mid u \in R \cap \mathcal{D}_{\Delta}\right\} .
$$

Proof. $(1 \Rightarrow 2)$ Suppose $\tau$ to be generated by an $\varepsilon$-CFTG $G=(N, \widehat{\Gamma}, \Sigma, S, P)$. We define the $\varepsilon$-CFG $G^{\prime}=\left(N, \widehat{\Delta}, S, P^{\prime}\right)$ where $\Delta=P$, and the set of productions of $P^{\prime}$ is obtained by transforming every $p: X \longrightarrow(\omega, v) \Omega(\bar{\omega}, w) \in P$ into $X \longrightarrow p \Omega \bar{p}$. Now, let $h: \widehat{\Delta}^{*} \rightarrow A^{*}$ and $g: \widehat{\Delta}^{*} \rightarrow \widehat{\Gamma}^{*}$ such that for every $p: X \longrightarrow(\omega, v) \Omega(\bar{\omega}, w) \in P, g(p)=\omega, g(\bar{p})=\bar{\omega}$ and $h(p)=v, h(\bar{p})=w$. Clearly we have

$$
\mathcal{T}_{G}=\left\{(g(u), h(u)) \mid u \in \mathcal{L}\left(G^{\prime}\right)\right\} .
$$

(2 $\Rightarrow 3$ ) Suppose that $\tau=\{(g(u), h(u)) \mid u \in X\}$ where $X$ is an $\varepsilon$-CFL and $g$ symmetric. From Theorem 8 , there is an alphabet $C$, a regular set $R \subseteq \widehat{C}^{*}$, and a symmetric homomorphism $g^{\prime}: \widehat{C}^{*} \rightarrow \widehat{\Delta}^{*}$ such that $X=g^{\prime}\left(R \cap \mathcal{D}_{\Delta}\right)$. The homomorphism $g \circ g^{\prime}$ is symmetric as $g$ and $g^{\prime}$ are both symmetric and $\tau=\left\{\left(g\left(g^{\prime}(x)\right), h\left(g^{\prime}(x)\right)\right) \mid x \in R \cap \mathcal{D}_{\Delta}\right\}$.

$(3 \Rightarrow 1)$ Let $\tau=\left\{(g(u), h(u)) \mid u \in R \cap \mathcal{D}_{\Delta}\right\}$ where $R$ is a regular language and $g$ is symmetric. From Proposition $5, R \cap \mathcal{D}_{\Delta}$ is an $\varepsilon$-CFL. Let us suppose that $R \cap \mathcal{D}_{\Delta}$ is generated by the $\varepsilon$-CFG $G=(N, \widehat{\Delta}, P, S)$, then $\tau$ is generated by the $\varepsilon$-CFTG $G^{\prime}=\left(N, \widehat{\Gamma}, \widehat{\Sigma} P^{\prime}, S\right)$ where

$$
P^{\prime}=\left\{X \longrightarrow(g(u), f(u)) \Omega(g(\bar{u}), h(\bar{u})) \mid X \longrightarrow u \Omega \bar{u} \in P, \Omega \in N^{*}, u \in \widehat{\Gamma}^{*}\right\} .
$$

Theorem 12 implies that the image of a set $X$ by an $\varepsilon$-CFT can be represented as $h\left(g^{-1}(X) \cap R \cap \mathcal{D}_{\Delta}\right)$ with $R$ being a regular set, $h$ a morphism and $g$ a symmetric morphism. It is then clear that the family of images of regular sets by $\varepsilon$-CFTs is the family of context-free languages; we will see (Theorem 15) that the family of images of the Dyck language is that of indexed languages, but more generally, images of $\varepsilon$-CFLs by $\varepsilon$-CFTs are recursively enumerable languages.

Proposition 13. Given a recursively enumerable language $E$, there is an $\varepsilon-C F T$ $\tau$, and an $\varepsilon-C F L Z$ such that $E=\tau(Z)$.

Proof. Let $E \subseteq \Sigma^{*}$. From [10], there is an alphabet $\Gamma$, a homomorphism $h$ : $\widehat{\Gamma}^{*} \rightarrow \Sigma^{*}$, and a context-free language $K \subseteq \widehat{\Gamma}^{*}$ such that $E=h\left(K \cap \mathcal{D}_{\Gamma}\right)$.

Let $g: \widehat{\Gamma}^{*} \rightarrow \widehat{\Gamma}^{*}$ be the injective symmetric homomorphism defined by $x \mapsto x \bar{x}$, for all $x \in \widehat{\Gamma}$. Then $E=h\left(g^{-1}(Z) \cap \mathcal{D}_{\Gamma}\right)$, for $Z=g(K)$, that is, from Theorem $12, E=\tau(Z)$, where $\tau$ is an $\varepsilon$-CFT. Note finally that $Z$ is an $\varepsilon$-CFL: from the grammar in Chomsky normal form generating $K$, one obtain an $\varepsilon$-CFG generating $Z$ by replacing the terminal productions $X \longrightarrow a$ by $X \longrightarrow g(a)$. 


\section{Characterizations of Indexed Languages}

In this final section, we relate indexed languages to $\varepsilon$-CFTs by showing that indexed language are sets $\tau\left(\mathcal{D}_{2}\right)$, where $\tau$ is an $\varepsilon$-CFT. This gives rise to various homomorphic characterizations of indexed languages.

\subsection{Indexed Grammars and Languages}

Introduced by Aho[1], indexed grammars extend context-free grammars by allowing nonterminals to yield a stack. Derivable elements are then represented by symbols $X^{\omega}$ where $X$ is a nonterminal and $\omega$ is a word called index word. Index words are accessed by a FIFO process: during a step of derivation of $X^{\omega}$, it is possible to add a symbol in head $\omega$, or to remove its first letter. Additionally, $\omega$ can be duplicated and distributed over other nonterminals.

Formally, an indexed grammar is a structure $\mathcal{J}=(N, I, \Sigma, S, P)$, where $N$ is the set of nonterminals, $\Sigma$ is the set of terminals, $S \in N$ is the start symbol, $I$ is a finite set of indexes, and $P$ is a finite set of productions of the form

$$
\begin{gathered}
X_{0}{ }^{\eta_{0}} \longrightarrow u_{0} X_{1}{ }^{\eta_{1}} u_{1} \cdots X_{n}{ }^{\eta_{n}} u_{n} \\
\text { with } u_{i} \in \Sigma^{*}, X_{i} \in N \text { and } \eta_{i} \in I \cup\{\varepsilon\} \text { for } i \in\{0, \ldots, n\} \text {. }
\end{gathered}
$$

Indexes are denoted as superscript, and we do not write indexes equal to $\varepsilon$.

Sentences are words $u_{1} A_{1}{ }^{\omega_{1}} \ldots u_{n} A_{n}{ }^{{ }^{\omega_{n}}} u_{n+1}$ with $u_{i} \in \Sigma^{*}, A_{i} \in N$ and $\omega_{i} \in I^{*}$. The derivation rule " $\longrightarrow \mathcal{J}$ " is a binary relation over sentences defined by

$$
\Omega_{1} A^{\eta \omega} \Omega_{2} \longrightarrow_{\mathcal{J}} \Omega_{1} u_{0} B_{1}{ }^{\eta_{1} \omega} \cdots B_{n}{ }^{\eta_{1} \omega} u_{n} \Omega_{2}
$$

iff there is a production $A^{\eta} \longrightarrow u_{0} B_{1}^{\eta_{1}} u_{1} \ldots B_{n}^{\eta_{n}} u_{n} \in P$.

The language generated by $\mathcal{J}$ is $\mathcal{L}_{\mathcal{J}}=\left\{u \in \Sigma^{*} \mid S \stackrel{*}{\longrightarrow}_{\mathcal{J}} u\right\}$. Languages generated by indexed grammars are called indexed languages.

Example 14. Let us consider the following indexed grammar $\mathcal{J}=(N, I, A, S, P)$ with $N=\{S, X, A, B, C\}, I=\{\beta, \alpha\}, A=\{a, b, c\}$ and $P$ consists of the following rules:

$$
\begin{array}{llll}
p_{1}: S \longrightarrow X^{\beta}, & p_{2}: S \longrightarrow \varepsilon, & p_{3}: X \longrightarrow X^{\alpha}, & p_{4}: X \longrightarrow A B C, \\
p_{5}: A^{\alpha} \longrightarrow a A, & p_{6}: A^{\beta} \longrightarrow \varepsilon, & p_{7}: B^{\alpha} \longrightarrow b B, & p_{8}: B^{\beta} \longrightarrow \varepsilon, \\
p_{9}: C^{\alpha} \longrightarrow c C, & p_{10}: C^{\beta} \longrightarrow \varepsilon . & &
\end{array}
$$

Here is a possible derivation:

$$
\begin{aligned}
S & \stackrel{p_{1}}{\longrightarrow} X_{\mathcal{J}} X^{\beta} \stackrel{p_{3}}{\longrightarrow} X^{\alpha \beta}{\stackrel{p_{3}}{\longrightarrow}}_{\mathfrak{J}} X^{\alpha \alpha \beta} \stackrel{p_{4}}{\longrightarrow} A^{\alpha \alpha \beta} B^{\alpha \alpha \beta} C^{\alpha \alpha \beta} \stackrel{p_{5}}{\longrightarrow} a A^{\alpha \beta} B^{\alpha \alpha \beta} C^{\alpha \alpha \beta} \\
& \stackrel{p_{5}}{\longrightarrow}{ }_{\mathfrak{J}} a a A^{\beta} B^{\alpha \alpha \beta} C^{\alpha \alpha \beta} \stackrel{p_{\mathfrak{G}}}{\longrightarrow} a a B^{\alpha \alpha \beta} C^{\alpha \alpha \beta} \stackrel{p_{7} p_{7} p_{8}}{\longrightarrow} a a b b C^{\alpha \alpha \beta} \stackrel{p_{9} p_{9} p_{10}}{\longrightarrow} a a b b c c
\end{aligned}
$$

The language generated by $\mathcal{J}$ is $\left\{a^{n} b^{n} c^{n}, n \geq 0\right\}$. 


\subsection{Characterizations of Indexed Languages}

We provide now homomorphic characterizations of indexed languages by establishing a strong connexion between indexed languages and $\varepsilon$-CFTs.

Theorem 15. A language $L$ is indexed iff there is an $\varepsilon-C F T \tau$ such that

$$
L=\tau\left(\mathcal{D}_{2}\right) .
$$

Let us informally explain the proof of Theorem 15. First we need to consider normal forms of indexed grammars (which extend the normal form given in [1]) and $\varepsilon$-CFT grammars.

An indexed grammar is said to be reduced if its productions are in the forms:

$$
\begin{gathered}
X_{0} \longrightarrow u X_{1}{ }^{\alpha} \cdots X_{n}{ }^{\alpha} v, \quad \text { or } X_{0}{ }^{\alpha} \longrightarrow u X_{1} \cdots X_{n} v ; \\
\text { with } n \geq 0, X_{i} \in N, u, v \in \Sigma^{*} \text { and } \alpha \in I \cup\{\varepsilon\} .
\end{gathered}
$$

An $\varepsilon$-CFTG is said to be reduced if its productions are in the form:

$$
X_{0} \longrightarrow(\alpha, u) \Omega(\bar{\alpha}, v) \text { with } \Omega \in N^{*}, u, v \in \Sigma^{*} \text { and } \alpha \in \widehat{\Gamma} \cup\{\varepsilon\} .
$$

Let us consider the bijective mapping $\varphi$ that maps a reduced indexed gram$\operatorname{mar} \mathcal{J}=(N, I, \Sigma, P, S)$ into a reduced $\varepsilon$-CFTG $\varphi(\mathcal{J})=(N, \widehat{I}, \Sigma, \varphi(P), S)$ by transforming every production

$$
\begin{aligned}
& p: X_{0} \longrightarrow u X_{1}{ }^{\alpha} \cdots X_{n}{ }^{\alpha} v \text { into } \varphi(p): X_{0} \longrightarrow(\alpha, u) X_{1} \cdots X_{n}(\bar{\alpha}, v), \text { and } \\
& p: X_{0}{ }^{\alpha} \longrightarrow u X_{1} \cdots X_{n} v \text { into } \varphi(p): X_{0} \longrightarrow(\bar{\alpha}, u) X_{1} \cdots X_{n}(\alpha, v) .
\end{aligned}
$$

The idea behind the construction is to write, into the terminal inputs of the $\varepsilon$-CFTG, the index operations made by the indexed grammar. The transduction grammar thus created is able to capture every index modifications of the initial indexed grammar, but also accepts bad computations. We claim that by restricting the domain to Dyck words, we exactly get derivations equivalent to those of the indexed grammar.

For example, there would be a derivation

$$
X \longrightarrow u_{1} X_{1}^{\alpha} v_{1} \longrightarrow u_{2} Y_{1}^{\alpha} Y_{2}{ }^{\alpha} v_{2} \longrightarrow u_{3} Y_{1}{ }^{\alpha} w_{3} Z v_{3}
$$

in $\mathcal{J}$ iff there was a derivation of the following form in $\varphi(\mathcal{J})$ :

$$
X \longrightarrow\left(\alpha, u_{1}\right) X_{1}\left(\bar{\alpha}, v_{1}\right) \longrightarrow\left(\alpha, u_{2}\right) Y_{1} Y_{2}\left(\bar{\alpha}, v_{2}\right) \longrightarrow\left(\alpha, u_{3}\right) Y_{1}\left(\bar{\alpha}, w_{3}\right) Z\left(\alpha \bar{\alpha}, v_{3}\right) .
$$

Claim: There is a derivation $S \stackrel{*_{\mathcal{J}}}{\longrightarrow} v_{1} Y_{1}^{w_{1}} v_{2} \cdots Y_{n}^{w_{n}} v_{n+1}$ iff there is a derivation $S \longrightarrow_{\varphi(\mathcal{J})}^{*}\left(u_{1}, v_{1}\right) Y_{1}\left(u_{2}, v_{2}\right) \cdots Y_{n}\left(u_{n+1}, v_{n+1}\right)$ where $u_{1} \cdots u_{n+1}$ belongs to $\mathcal{D}_{I}$ and $\rho\left(u_{1} \cdots u_{i}\right)=w_{i}^{R}$ for $i \in\{1, \ldots, n\}$ ( $w_{i}^{R}$ is the mirror image of $\left.w_{i}\right)$.

This can be proved by induction over the length of derivations, and implies that $\mathcal{T}_{\varphi(\mathcal{J})}\left(\mathcal{D}_{I}\right)=\mathcal{L}_{\mathcal{J}}$. Because of the bijectivity of the construction, we obtain:

"A language $L$ is indexed iff there is an $\varepsilon$-CFT $\tau$ and $k \in \mathbb{N}$ s.t. $L=\tau\left(\mathcal{D}_{k}\right)$."

Finally, it is possible to define from every $\varepsilon$-CFT $\tau$, an $\varepsilon$-CFT $\tau^{\prime}$ such that $\tau\left(\mathcal{D}_{\Gamma}\right)=\tau^{\prime}\left(\mathcal{D}_{2}\right)$, by encoding every $\alpha_{i} \in \Gamma$ by a word $01^{i} 0$ and $\bar{\alpha}_{i}$ by $\overline{0} \overline{1}^{i} \overline{0}$. 
Example 16. Let $\mathcal{J}=(N, I, \Sigma, S, P)$ be an indexed grammar with $N=\{S, X, Y$, $W, Z\}, I=\{\beta, \alpha\}, A=\{a\}$ and $P$ consists of the rules:

$$
\begin{aligned}
& S \longrightarrow X^{\beta}, \quad X \longrightarrow X^{\alpha}, \quad X \longrightarrow Y, \quad Y^{\alpha} \longrightarrow a Y Z \\
& Y^{\beta} \longrightarrow \varepsilon, \quad Z^{\alpha} \longrightarrow a Z a, \quad Z^{\beta} \longrightarrow \varepsilon .
\end{aligned}
$$

Initially defined in [7], the grammar $\mathcal{J}$ generates the language $L=\left\{a^{n^{2}} \mid n \geq 0\right\}$. Applying the bijection $\varphi$ defined above to J, we get the $\varepsilon$-CFTG $G$ given in Example 11 and generating the transduction

$$
\tau=\bigcup_{n, m, r_{1}, \ldots . r_{m} \geq 0}\left(\beta \alpha^{n} \bar{\alpha}^{m} \bar{\beta} \beta\left(\Pi_{i=1}^{m} \bar{\alpha}^{r_{i}} \bar{\beta} \beta \alpha^{r_{i}+1}\right) \bar{\alpha}^{n} \bar{\beta}, a^{m+2 r_{1}+\cdots+2 r_{m}}\right) .
$$

For every $u=\beta \alpha^{n} \bar{\alpha}^{m} \bar{\beta} \beta\left(\Pi_{i=1}^{m} \bar{\alpha}^{r_{i}} \bar{\beta} \beta \alpha^{r_{i}+1}\right) \bar{\alpha}^{n} \bar{\beta} \in \operatorname{Dom}(\tau)$,

$u$ is a Dyck word $\Longrightarrow m=n, r_{1}=0$, and for all $i \in[0, m-1], r_{i+1}=r_{i}+1$

$$
\begin{aligned}
& \Longrightarrow \tau(u)=a^{n+2(0+1+\cdots+n-1)} \\
& \Longrightarrow \tau(u)=a^{n^{2}} .
\end{aligned}
$$

It follows that $\tau\left(\mathcal{D}_{I}\right)=\left\{a^{n^{2}}\right\}_{n \geq 0}=\mathcal{L}_{\mathcal{J}}$.

Corollary 17. A language $L$ is indexed if there is a homomorphism h, a symmetric homomorphism $g$, a regular set $R$ and $k \in \mathbb{N}$ such that

$$
L=h\left(g^{-1}\left(\mathcal{D}_{2}\right) \cap R \cap \mathcal{D}_{k}\right) .
$$

Theorem 18. A language $L$ is indexed iff there is an $\varepsilon$-CFL $K$, a morphism $h$, and an alphabet $\Gamma$ such that

$$
L=h\left(K \cap \mathcal{D}_{\Gamma}\right) .
$$

Proof. $(\Rightarrow)$ Let $L \subseteq A^{*}$ be an indexed language. From Theorem 15 and Theorem 12 , there are alphabets $\Sigma, \Gamma$, an $\varepsilon$-CFL $K \subseteq \widehat{\Gamma}^{*}$, a homomorphism $h: \widehat{\Gamma^{*}} \rightarrow A^{*}$ and a symmetric homomorphism $g: \widehat{\Gamma}^{*} \rightarrow \widehat{\Sigma}^{*}$ such that $L=h\left(K \cap g^{-1}\left(\mathcal{D}_{\Sigma}\right)\right)$. We suppose that $\Sigma \cap A=\emptyset$ (otherwise, it suffices to work with a copy of $\Sigma$ ), and define the homomorphism $\mu: \widehat{\Gamma}^{*} \rightarrow \widehat{\Delta}^{*}$, for $\Delta=\Sigma \cup A$, by $\alpha \mapsto g(\alpha) h(\alpha) \overline{h(\alpha)}$. For all $u \in \widehat{\Gamma}^{*}, \mu(u) \in \mathcal{D}_{\Delta}$ iff $u \in g^{-1}\left(\mathcal{D}_{\Sigma}\right)$; in addition, $\pi_{A}(\mu(u))=h(u)$, with $\pi_{A}$ being the projection of $\widehat{\Delta}^{*}$ into $A^{*}$. Then we have:

$$
\pi_{A}\left(\mu(K) \cap \mathcal{D}_{\Delta}\right)=h\left(K \cap g^{-1}\left(\mathcal{D}_{\Sigma}\right)\right)=L .
$$

Now, as $\mu$ satisfies " $\rho(u)=\varepsilon \Longrightarrow \rho(g(u))=\varepsilon$ " and $K$ is an $\varepsilon$-CFL, so is $\mu(K)$. $(\Leftarrow)$ Obvious from Theorem 15 and Proposition 12, by choosing $g$ to be the identity mapping.

\section{Acknowledgement}

The authors would like to thank Pr. Jean-Marc Talbot whose remarks and suggestions greatly improved the development of this paper. 


\section{References}

1. Aho, A.: Indexed grammars-an extension of context-free grammars. J. ACM 15, 647-671 (1968)

2. Berstel, J.: Transductions and Context-Free Languages. Teubner Verlag (1979)

3. Berstel, J., Boasson, L.: In: Handbook of Theoretical Computer Science (Vol. B), chap. Context-free Languages, pp. 59-102. MIT Press (1990)

4. Berstel, J., Boasson, L.: Balanced grammars and their languages. In: Formal and Natural Computing. Lecture Notes in Comput. Sci., vol. 2300, pp. 3-25. Springer (2002)

5. Caucal, D.: On infinite transition graphs having a decidable monadic theory. Theor. Comput. Sci. 290(1), 79-115 (2003)

6. Chomsky, N., Schützenberger, M.P.: The algebraic theory of context-free languages. In: Computer Programming and Formal Systems, pp. 118-161. Studies in Logic, North-Holland Publishing (1963)

7. Fischer, M.J.: Grammars with macro-like productions. In: 9th Annual Symposium on Switching and Automata Theory. pp. 131-142. IEEE Computer Society (1968)

8. Fratani, S.: Automates à piles de piles ... de piles. Ph.D. thesis, Université Bordeaux $1(2005)$

9. Hague, M., Murawski, A.S., Ong, C.L., Serre, O.: Collapsible pushdown automata and recursion schemes. In: LICS, Proceedings. pp. 452-461. IEEE Computer Society $(2008)$

10. Hirose, S., Nasu, M.: Left universal context-free grammars and homomorphic characterizations of languages. Information and Control 50(2), 110 - 118 (1981)

11. Kanazawa, M.: Multidimensional trees and a Chomsky-Schützenberger-Weir representation theorem for simple context-free tree grammars. J. of Logic and Computation (2014)

12. Knapik, T., Niwinski, D., Urzyczyn, P.: Higher-order pushdown trees are easy. In: FOSSACS. Lecture Notes in Comput. Sci., vol. 2303, pp. 205-222. Springer (2002)

13. Maslov, A.N.: Hierarchy of indexed languages of arbitrary level. Soviet Math. Dokl 115(14), 1170-1174 (1974)

14. Maslov, A.N.: Multilevel stack automata. Problems of Information Transmission 12, 38-43 (1976)

15. Mehlhorn, K.: Pebbling mountain ranges and its application to DCFL-recognition. Automata, Languages and Programming 85, 422-435 (1980)

16. Nivat, M.: Transductions des langages de Chomsky. Ann. Inst. Fourier 18(1), 339 455 (1968)

17. Okhotin, A.: Non-erasing variants of the chomsky-schützenberger theorem. In: Developments in Language Theory. Lecture Notes in Comput. Sci., vol. 7410, pp. 121-129. Springer (2012)

18. Ong, L.: Higher-order model checking: An overview. In: LICS. pp. 1-15. IEEE Computer Society (2015)

19. Sorokin, A.: Monoid automata for displacement context-free languages. CoRR abs/1403.6060 (2014)

20. Weir, D.: Characterizing Mildly Context-Sensitive Grammar Formalisms. Ph.D. thesis, University of Pennsylvania (1988), available as Technical Report MS-CIS88-74 